\title{
Synthesis, Growth and Characterization of Semi Organic Nonlinear Optical Maleic Added Lithium Sulphate Monohydrate Crystal
}

\author{
G. Theophil Anand*, D. Suganya \\ Department of Physics and Abraham Panampara Research Centre, Sacred Heart College (Autonomous), Tirupattur - 635 601, Tamil Nadu, India.
}

\section{ART ICLE DETAILS}

Article history:

Received 21 February 2018

Accepted 10 March 2018

Available online 14 March 2018

Keywords:

Solution Growth

Optical Analysis

NLO Properties

\begin{abstract}
A B S T R A C T
The present study aims at exploring the enhancing effects of maleic acid on the lithium sulphate monohydrate crystals grown by slow evaporation method. The powder XRD analysis confirm that the grown crystal belong to monoclinic structure with space group $\mathrm{P} 2_{1}$. The Fourier transform infrared spectroscopy study revealed the inclusion of the maleic acid into the crystal. The optical transmittance study was used to estimate the lower cut off frequency and optical band gap. The Vicker's microhardness test was employed to find out the mechanical strength of the crystal.
\end{abstract}

\section{Introduction}

Lithium sulphate monohydrate is an important material, with extremely large coefficient, used as hydrostatic pressure sensors [1]. The structure of lithium sulphate monohydrate was investigated as monoclinic and space group $\mathrm{P} 2{ }_{1}$ by Ziegler [2], which contains the $\mathrm{Li}^{+}$cations, tetrahedral $\mathrm{SO}_{4}{ }^{2-}$ anions and water molecule. Ionic bonds between cations and anions extend in all directions in the crystals but the hydrogen bonding between water molecules extend only along $b$, the unique polar axis [1]. This anisotropic structure of the material enables large polarizibility, ferroelectric, piezoelectric [3] and pyroelecrtric property [4]. LSM was identified as a NLO active material [5] and also explored enhanced NLO efficiency when combined with glycine and selenate $[6,7]$. It was found from the study that the LSMH crystals were proved to be optically improved transmittance, mechanically harder and thermally stable when doped with EDTA and $\mathrm{Cu}(\mathrm{II})[8,9]$. The need of high quality non-linear optical (NLO) material for hi-tech components has enforced many researchers to modify the intrinsic properties of already grown crystals by inclusion of acid additives. The organic substituent offer better photochemical stability and more acentric molecular alignment to host material. Maleic acid (MA) belong to the family of carboxylic acids that might offer wide bonding network for itinerancy of p-electrons which is the foremost requirement for improving the NLO properties and the electronic response time of single crystal. Recent research discovered notable SHG efficiency enhancement in KDP single crystal by using carboxylic acids (FA, OA, MA) as additives for the applications of NLO facilitated photonic devices $[10,11]$. In this fashion, the present investigation is also an attempt to closely scrutinize the significant role maleic acid in uplifting the qualities of lithium sulphate monohydrate.

After reviewing many articles regarding single crystal grown by slow evaporation method which has NLO properties, and especially the last three articles mentioned in the literature survey has given vigor to do a project in lithium sulphate monohydrate and maleic acid. Besides, the synthesis of lithium sulphate monohydrate oxalate gives a clear idea about the semi-organic materials for NLO applications. As well as it is obvious that lithium sulphate monohydrate (LSMH) is an inorganic material and has a NLO property and maleic acid is an organic material and when both are combined together it forms as a semi-organic material for an enhanced NLO properties. Hence the work focuses on the preparation of pure lithium sulphate monohydrate (LSMH) and maleic added lithium sulphate monohydrate (MLSMH) and comparison of the same for NLO applications.

\section{Experimental Methods \\ 2.1 Materials}

Lithium sulphate monoydrate (Merck) (99\% AR grade), maleic acid (Merck) (99\%AR grade), $\mathrm{NaOH}$ and de-ionized water.

\subsection{Synthesis of Pure LSMH}

The single crystal of the pure LSMH was grown by slow evaporation method at room temperature. The calculated amount of LSMH and $20 \mathrm{~mL}$ of deionized water are dissolved well using magnetic stirrer to get a saturated solution. The saturated solution were filtered and covered with porous polythene sheet. The solution was allowed to evaporate at room temperature, after 4 weeks, the single crystal were grown and it is shown in Fig. 1.

\subsection{Synthesis of Maleic Added Lithium Sulphate Mono Hydrate (MLSMH)}

The single crystal of MLSMH was grown by slow evaporation method at room temperature. The saturated solution MLSMH was prepared as follows. $10 \mathrm{~mL}$ of deionized water was taken. Lithium sulphate monohydrate (LSMH) was slowly dissolved in $10 \mathrm{~mL}$ of deionized water with continuous stirring to get a saturated solution then maleic was slowly dissolved in $10 \mathrm{~mL}$ of deionized water to get a saturated solution. Both the solution added and it were dissolved well for 30 minutes to get a saturated solution. After that the $\mathrm{pH}$ of the saturated solution was adjusted to 4.3, which is one unit greater than that of the pKa value using $\mathrm{NaOH}$ to stimulate the dissociation of the compound. The solution was filtered, transferred into beaker and covered it with porous polythene sheet. The solution was allowed to evaporate at room temperature. After a few days MLSMH single crystal were grown in the bottom of the beaker. It is shown in the Fig. 1.

\subsection{Characterization of Maleic Added Pure LSMH}

Structural analysis were confirmed by X-Ray powder diffraction (XRD), the presence the vibrational modes were identified using Fourier transform infrared spectrometry (FT-IR), UV-Visible spectrum confirm the absorption of grown crystal. The stability of the material was found by thermo gravimetric analysis (TGA) and differential thermal analysis (DTA) technique. The mechanical strength of the material was confirmed with the help of Vickers's microhardness tester. The powder SHG technique confirms the presence second harmonic generation of the material. 


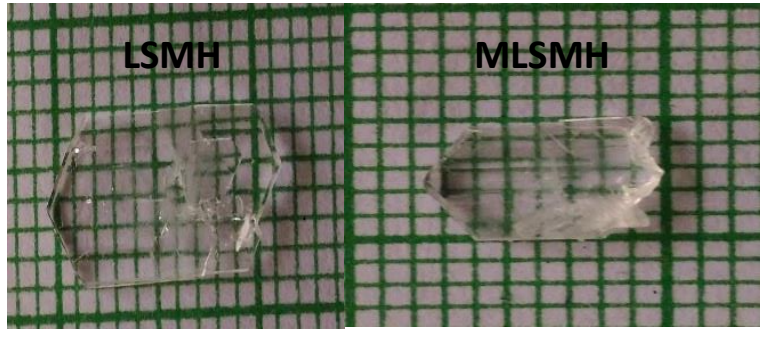

Fig. 1 Photograph of LSMH and MLSMH

\section{Results and Discussion}

\subsection{Powder XRD Analysis of Pure LSMH and MLSMH}

The crystalline quality of the grown crystal was determined by powder X-ray diffraction (XRD) technique using a $\mathrm{Cu} K \alpha$ source $(\lambda=1.5405 \AA$ ). Fig. 2 depicts the PXRD patterns of the diffracted crystal. The sharp diffraction peaks in the pattern advocates improved crystalline quality. The plane corresponding to the peaks were identified using the software Powder X. The notable observations are, all atoms in the pure LSM crystals are oriented along the plane 101 , whereas in the maleic acid added LSMH crystals along 111 and also gauged reduction in the intensity of the same plane relative to the pure. The crystal structure was maintained to be monoclinic with space group $\mathrm{P} 22_{1}$. The modification in the intensity of the peaks and the appearance of additional faces confirm the maleic acid substitution in the LSM structure. The evaluated cell parameters are $a=$ $7.1397 \AA \quad b=7.1397 \AA \quad c=10.6121 \AA$. The datum inferred from powder XRD analysis are given in the Table. The slight shift in the sharp peak position at $25.3^{\circ}$ in lower angle side may.

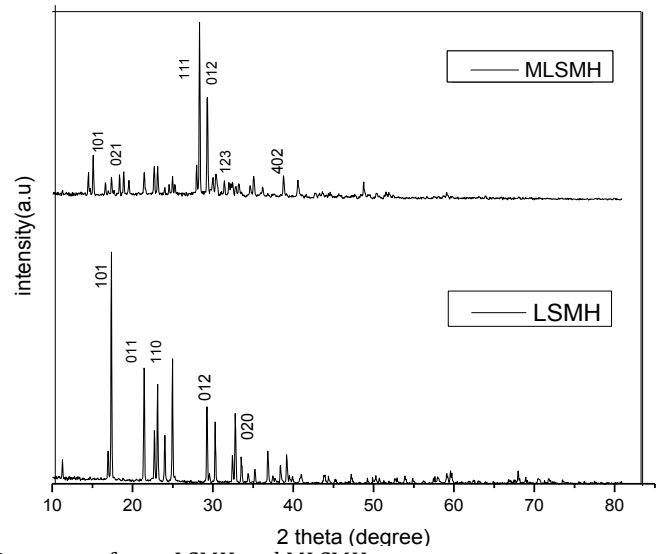

Fig. 2 XRD pattern of pure LSMH and MLSMH
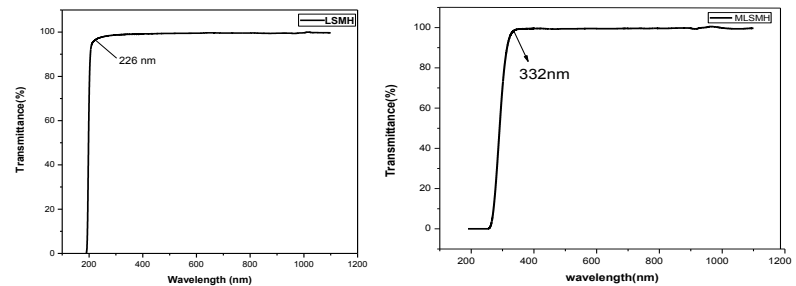

Fig. 3 UV-vis spectrum of Pure LSMH and maleic acid added MLSMH

\subsection{UV-Visible Spectral Analysis of Pure LSMH and MLSMH}

The optical properties of the maleic acid added lithium sulphate monohydrate were evaluated in the wavelength region $190 \mathrm{~nm}$ to 1100 nm using an ultraviolet-visible (UV-vis) spectrometer (Perkin Elmer Lambda 35). The cut off wavelength of pure LSMH is found to be at $226 \mathrm{~nm}$ and the inspection of Fig. 3 yields the lower cut off wavelength and optical band gap as $332 \mathrm{~nm}$ and $3.74 \mathrm{eV}$ respectively. Fig. 3 represents the transmittance as a function of the wavelength. As there is no absorption in the visible and near infra-red region it can be used as a potential material for frequency doubling [12]. It is noticeable that the addition of maleic acid has appreciably modified the transmittance cut-off wavelength of LSMH crystal. The $100 \%$ transmittance of MLSMH crystal in the entire visible region reveals that the inclusion of maleic acid might have significantly reduced the solvent inclusions and defect centers responsible for optical scattering within the crystal system eventually resulting in enhancement optical property of the grown crystal. The MLSMH crystals with high transmittance and modified cut-off wavelength may find huge advantage in UV-vis-tunable lasers and NLO device applications $[13,14]$.

\subsection{FTIR Analysis for Pure LSMH and MLSMH}

The qualitative analysis of the grown crystal has been done from the FTIR spectrum recorded by using Perkin Elmer Spectrum RX I spectrometer operative in the wavelength region $400-4000 \mathrm{~cm}^{-1}$. The output of the LSMH FTIR spectrometer is shown in Fig. 4. A perusal of the FTIR spectrum of LSMH accredit to symmetric and asymmetric stretching of carboxylate anion at wave number $1613 \mathrm{~cm}^{-1}$. The band at $998 \mathrm{~cm}^{-1}$ is due to $\mathrm{O}-\mathrm{H}$ deformation and the absorption at $578 \mathrm{~cm}^{-1}$ is assigned to $\mathrm{C}-\mathrm{O}$ deformation [15]. The peak at $3499 \mathrm{~cm}^{-1}$ is attributed to the presence of water molecules in the grown crystal. The characteristic vibration of $\mathrm{SO}_{4}{ }^{2-}$ group appears at $1116 \mathrm{~cm}^{-1}$ and $639 \mathrm{~cm}^{-1}$ [16]. The output of the FTIR spectrometer is shown in Fig. 4. A perusal of the FTIR spectrum of MLSMH accredit to symmetric and asymmetric stretching of carboxylate anion at wave number $1654 \mathrm{~cm}^{-1}$ and $1387 \mathrm{~cm}^{-1}$ respectively. The band at $864 \mathrm{~cm}$ 1 is due to $0-\mathrm{H}$ deformation and the absorption at $567 \mathrm{~cm}^{-1}$ is assigned to C-O deformation [15]. The peak at $3509 \mathrm{~cm}^{-1}$ is attributed to the presence of water molecules in the grown crystal. The characteristic vibrations of $\mathrm{SO}_{4^{-}}$group appears at $1100 \mathrm{~cm}^{-1}, 864 \mathrm{~cm}^{-1}, 639 \mathrm{~cm}^{-1}$ [16].
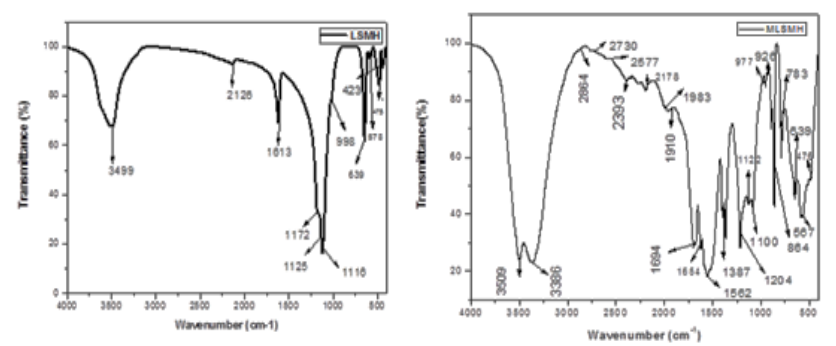

Fig. 4 FTIR spectra of LSHM and MLSMH

\subsection{Thermal Analysis (TGA and DTA) of Pure LSMH and MLSMH}

TGA and DTA studies of pure LSMH and MLSMH crystal were carried out between $30^{\circ} \mathrm{C}$ and $700{ }^{\circ} \mathrm{C}$ in nitrogen atmosphere using Perkin-Elmer diamond analyser. The TGA and DTA curve of LSMH and MLSMH crystals were shown in the Fig. 5 . The water molecules will be evaporated the temperature of $103^{\circ} \mathrm{C}$ of LSMH. The phase transition takes place from 103 ${ }^{\circ} \mathrm{C}$ up to $127{ }^{\circ} \mathrm{C}$. From the DTA curve, there is one exothermic and one endothermic peak was observed in LSMH. In TGA and DTA curve, the water molecules will be evaporated at the temperature of $106{ }^{\circ} \mathrm{C}$ of MLSMH crystal. The phase transition takes places form $106^{\circ} \mathrm{C}$ to $126^{\circ} \mathrm{C}$. From the DTA curve, there is one exothermic and one endothermic peak was observed in MLSMH.
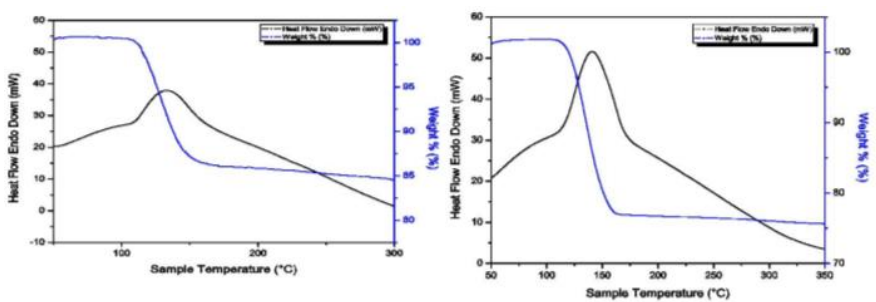

Fig. 5 TGA \& DTA of pure LSMH maleic acid added MLSMH

\subsection{Vicker's Microhardness of Pure LSMH and MLSMH}

The hardness is a property of the materials ability to resist wear, abrasion, cutting and indentation. This may be uniform throughout the material. It is often measured using its resistance to the indentation. Increase in hardness would also increase the brittleness of the material. The Vicker's hardness studies have been performed on maleic acid added lithium sulphate monohydrate crystal using the Shimadzu HMV-2T micro hardness analyzer. The hardness strength of grown crystal was evaluated by applying load of 25, 50 and $100 \mathrm{~g}$ for constant indentation duration of $6 \mathrm{~s}$ for all loads. The microhardness number (VHN) was calculated using formula, $\mathrm{H}_{\mathrm{v}}=1.8544\left(\mathrm{P} / \mathrm{d}^{2}\right) \mathrm{Kg} / \mathrm{mm}^{-2}$ Where, $\mathrm{P}$ is the applied load $(\mathrm{g})$ and $\mathrm{d}$ is the average diagonal length $(\mathrm{mm})$ of the indentation mark. The resist deformation under the action of loads shown in Fig. 6 clearly indicates that the microhardness number increases with increase in load satisfying the reverse indentation size effect theory. By using the Mayer's law $\mathrm{P}=\mathrm{adn}$ that relates applied load $\mathrm{P}$ and diagonal length $\mathrm{d}$, the Mayer's index (work hardening coefficient) $\mathrm{n}$ was calculated as 5.2 . Hence it belongs to soft 
material category. The hardness parameters are most decisive prerequisites for avoiding breakage/wastage of material during polishing and processing the crystals to exploit it to device fabrication [17].

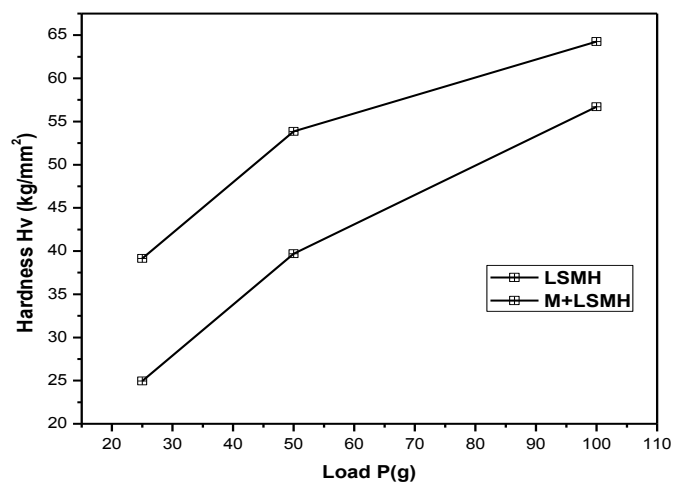

Fig. 6 Load vs. hardness number of Pure LSMH and MLSMH

\subsection{Second Harmonic Generation studies (Nonlinear Optical Property)}

The second harmonic generation efficiency was estimated by powder technique of Kurtz and Perry [12]. The powder sample of maleic acid added lithium sulphate monohydrate LSMH) was irradiated with the incident radiation of wavelength $1064 \mathrm{~nm}$ from Nd: YAG laser source. The result indicates that the powder SHG efficiency of MLSMH is improved to 1.1 times higher than that of KDP.

\section{Conclusion}

The influence on the maleic acid added lithium sulphate monohydrate (MLSMH) crystal was grown by slow solution evaporation technique. The monoclinic crystal structure and change in the lattice parameters value confirm the inclusion of maleic acid into the grown crystals. The prominent peaks of the maleic acid and lithium sulphate monohydrate were successfully identified in FTIR spectrum. The UV-vis spectrum analysis revealed the transparency of the crystal in the visible region. The Mayer's index (n) was calculated as 5.2 and observed increase in Vicker's micro hardness value with respect to load.

\section{References}

[1] R.E. Newnham, D.P. Skinner, Connectivity and piezoelectric-Pyroelectric composites, Mat. Res. Bull. 13(5) (2013) 525-536.

[2] G.E. Ziegler, The crystal structure of lithium sulphate mono-hydrate, New York Univ. Bobst Lib. 1 (1934) 456-461

[3] M. Anis, G.G. Muley, M.D. Shirsat, S.S. Hussaini, Single crystal growth, structural, optical, mechanical, dielectric and thermal studies of formic acid doped potassium dihydrogen phosphate crystal for NLO applications, Cryst. Res. Technol. 50(5) (2015) 372-378.

[4] Sidney B. Lang, Pyroelectric coefficient of lithium sulfate monohydrate (4.2320 K), Phys. Rev. B 4(10) (1971) 3603-3609.

[5] L. Bayajargal, Nonlinear optical properties of lithium sulfate monohydrate, Cryst. Res. Technol. 43 (2008) 1138-1142.

[6] T. Balakrishnan, K. Ramamurthi, Growth and characterization of glysine lithium sulphate single crystal, Cryst. Res. Technol. 41 (2006) 1184-1188.

[7] R. Frech, E. Cazzanelli, Vibrational study of selenate-doped lithium sulphate:single crystal and fused salts, J. Solid State Chem. 74 (1988) 256-259.

[8] R. Manimekalai, A. Pushal Raj, C. Ramachandra Raja, Growth and characterization of ethylene diamine tetra acetate doped of lithium sulphate monohydrate crystals, Opt. Photon. Jour. 2 (2012) 216-221.

[9] K. Boopathi, P. Ramasamy, G. Bhagavannarayana, Growth and characterization of $\mathrm{Cu}$ (II) doped negatively soluableof lithium sulphate monohydrate crystals, Jour. Cryst. Growth 386 (2014) 32-37.

[10] Mohd Anis, G.G. Muley, A. Hakeem, M.D. Shirsat, S.S. Hussaini, Exploring the influence of carboxylic acids on nonlinear optical (NLO) and dielectric properties of KDP crystal for applications of NLO facilitated photonic devices, Opt. Mater. 46 (2015) 517-521.

[11] Mohd Anis, G.G. Muley, M.I. Baig, S.S. Hussaini, M.D. Shirsat, Doping effect of carboxylic acids on optical, electrical, mechanical and thermal traits of KDP crystal, Mater. Res. Innovat. 21 (2016) 439-446.

[12] T. Balakrishnan, K. Ramamurthi, Growth and characterization of glycine lithium sulphate single crystal, Cryst. Res. Technol. 40(12) (2006) 1184-1188.

[13] M. Anis, D.A. Hakeem, G.G. Muley, Optical and dielectric studies of $\mathrm{KH}_{2} \mathrm{PO}$ crystal influenced by organic ligand of citric acid and L-valine: A single crystal growth and comparative study, Result. Phys. 6 (2016) 645-650.

[14] N.N. Shejwal, Mohd Anis, S.S. Hussainiz, M.D. Shirsat, Investigation on structural, UV-visible, SHG efficiency, dielectric, mechanical and thermal behavior of L-cystine doped zinc thiourea sulphate crystal for NLO device applications, Int. J. Modern Phys. B 30 (2016) 1650159-1650169.

[15] M. Lakshmipriya, R. Ezhil Vizhi, D. Rajan Babu, Growth and characterization of maleic acid single crystal, Optik 126 (2015) 4259-4262.

[16] K. Boopathi, P. Ramasamy, G. Bhagavannarayana, Growth and characterization of $\mathrm{Cu}$ (II) doped negatively soluble lithium sulphate monohydrate crystals, Jour. Cryst. Growth 386 (2014) 32-37.

[17] Y.B. Rasal, M. Anis, M.D. Shirsat, S.S. Hussaini, Growth, structural, UV-visible, SHG, mechanical and dielectric studies of bis-thiourea zinc chloride doped KDP crystal for NLO device applications, Mater. Res. Innovat. 21 (2016) 45-49. 